\title{
Discrepancies among clinical, histological and immunological findings in IgA pemphigus: a case report and literature survey
}

\author{
Kaja Męcińska-Jundziłł¹, Takashi Hashimoto², Cezary Kowalewski ${ }^{3}$, Katarzyna Woźniak ${ }^{3}$, Norito Ishii ${ }^{4}$, \\ Rafat Czajkowski ${ }^{1}$ \\ ${ }^{1}$ Chair of Dermatology, Sexually Transmitted Diseases and Immunodermatology, Nicolaus Copernicus University in Torun, \\ Faculty of Medicine, Bydgoszcz, Poland \\ ${ }^{2}$ Institute of Cutaneous Cell Biology, Kurume University, Fukuoka, Japan \\ ${ }^{3}$ Department of Dermatology and Immunodermatology, Medical University of Warsaw, Warsaw, Poland \\ ${ }^{4}$ Department of Dermatology, School of Medicine, Kurume University, Fukuoka, Japan
}

Adv Dermatol Allergol 2016; XXXIII (6): 480-484

DOI:10.5114/pdia.2016.62486

IgA pemphigus is a rare variant of pemphigus presented clinically with vesiculopustular skin lesions, and histopathologically neutrophil infiltration and acantholysis in the epidermis. Moreover, circulating IgA autoantibodies target cell surface components of the epidermis.

According to clinical, histopathological and direct immunofluorescence features, IgA pemphigus is subdivided into subcorneal pustular dermatosis (SPD) type reactive with desmocollin 1 (Dsc1) [1] and intraepidermal neutrophilic IgA dermatosis (IEN) type reactive with an unknown antigen. However, in some reported cases of IEN type IgA pemphigus, the IgA anti-cell surface autoantibodies reacted with desmoglein 1 (Dsg1) or Dsg3 [2, $3]$, which are widely accepted to be the target antigens in pemphigus foliaceus and pemphigus vulgaris, respectively.

Here, we present a case of IgA pemphigus with histopathological features typical of the SPD type and immunological characteristics of the IEN type with anti-Dsg3 antibodies, and a review of the literature.

An 87-year-old male of Polish origin presented erosions in the oral mucosa and in intertriginous areas. The patient had numerous internal disorders, including ischemic cardiomyopathy, type 2 diabetes, abdominal aortic aneurysm, partial stomach resection because of peptic ulcer disease, colitis, diverticular disease, iron deficiency anemia, hemorrhoids and prostatic hypertrophy, and took torasemide, finasteride, acetylsalicylic acid, pantoprazole, ramipril and nebivolol.
The patient was initially treated in the Department of Internal Medicine with topical antibiotics and topical glucocorticosteroids with no improvement. Oral prednisolone in a dose of $10 \mathrm{mg} /$ day for 2 weeks was not effective, either. The unsuccessful treatment and polymorphic skin lesions led to the hypothesis of paraneoplastic disorder. Therefore, abdominal ultrasonography, gastroscopy and chest X-ray were performed and showed no abnormalities. However, the fecal blood test was positive in the patient and colonoscopy evaluation revealed colitis. Histopathological evaluation of the patient skin indicated pyodermitis, whereas the results of direct and indirect immunofluorescence were negative.

Three months later, the patient was referred to the Department of Dermatology. Physical examination revealed annular erythematous skin lesions with erosions and pustules on the abdomen, groins, buttocks and right armpit. Groins were most severely affected (Figure 1). Erosive lesions of the oral mucosae in the anamnesis were already healed at that time. Laboratory examinations showed microcytic anemia, elevated C-reactive protein (40.91 mg/l; normal < $5 \mathrm{mg} / \mathrm{l})$, low level of sodium (133.8 mmol/l; normal $136 \mathrm{mmol} / \mathrm{l}$ ) and extremely lowered serum albumins (1.93 g/dl; normal $3.5 \mathrm{~g} / \mathrm{dl})$.

Histopathology of skin lesion revealed extensive neutrophilic pustules in the upper part of the epidermis, with minimum pustules in the middle epidermis (Figure 2 A). Direct immunofluorescence of perilesional skin disclosed IgA deposits on cell surfaces of the entire

Address for correspondence: Kaja Męcińska-Jundziłł MD, Chair of Dermatology, Sexually Transmitted Diseases and Immunodermatology, Faculty of Medicine, Nicolaus Copernicus University, 9 Sklodowskiej-Curie St, 85-094 Bydgoszcz, Poland, phone: +48 602404829 , e-mail: kaja_cm@wp.pl

Received: 18.08 .2016 , accepted: 20.09.2016 


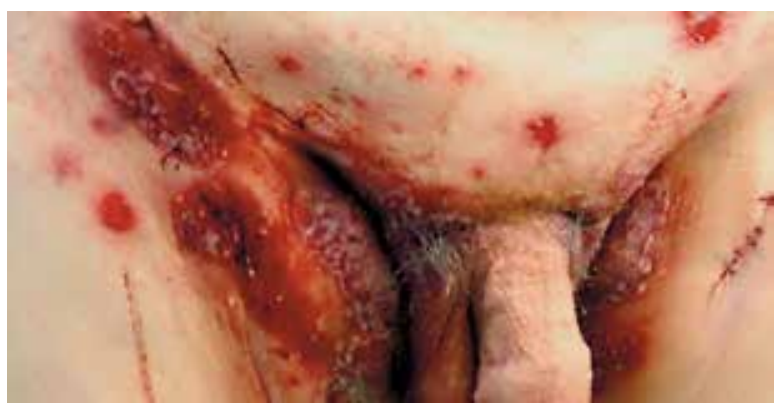

epidermis (Figure 2 B). Indirect immunofluorescence of monkey esophagus showed IgA, but not IgG, anti-cell surface autoantibodies at a titer of $1: 160$ (Figure 2 C).

Then, to define the subtype of IgA pemphigus, we performed further immunological studies. Immunoblotting of normal human epidermal extract showed negative results for both IgG and IgA antibodies. IgA ELISAs of recombinant baculoprotein (RPs) of Dsgs revealed positive reactivity with Dsg3 (optical density $(O D)=1.063$, cut-off $>0.15$ ), but negative reactivity with Dsg1 (OD $=0.006$, cut-off > 0.15). IgG ELISA of Dsg1 and Dsg3 showed negative results. Novel ELISAs of mammalian RPS of Dsc1-3 showed negative results for both IgG and IgA antibodies.

Dapsone of $100 \mathrm{mg} /$ day led to a significant improvement within few days. Although the dosage was reduced
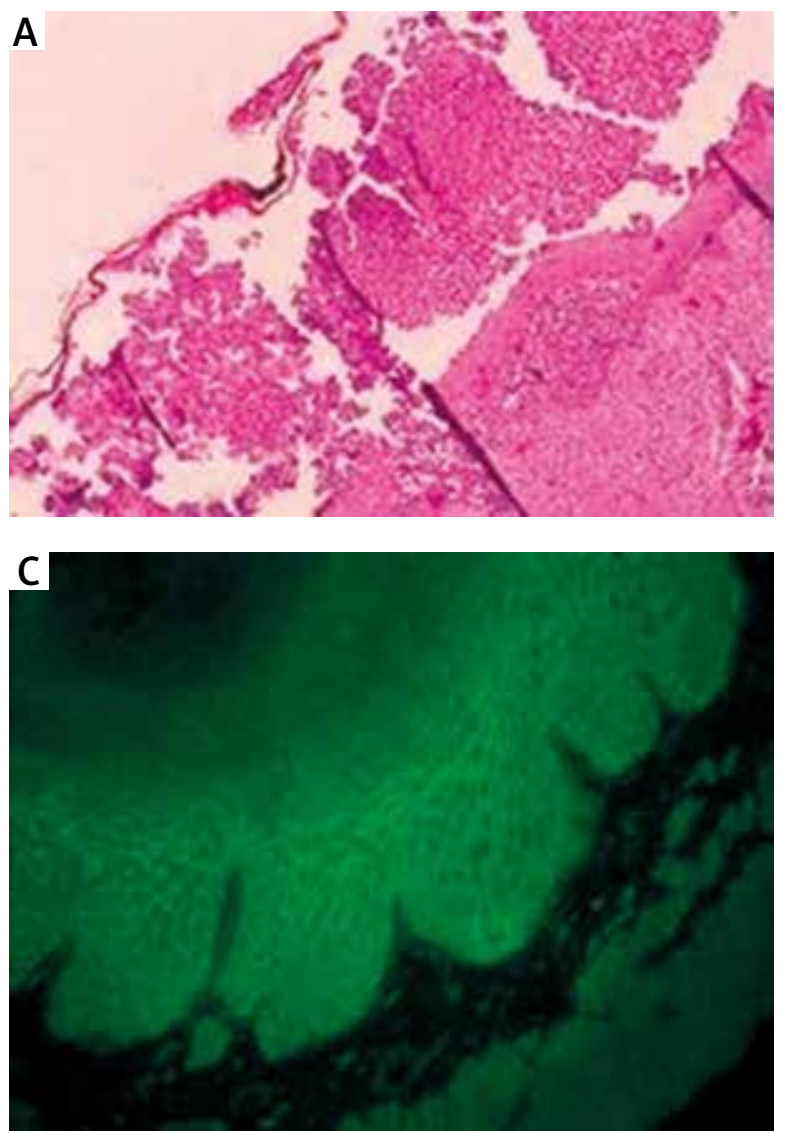

Figure 1. Annular erythematous skin lesions with erosions and pustules within groins

to $50 \mathrm{mg} /$ day because of methemoglobinemia $(0.50 \%$ $1.20 \%-1.80 \%$ ) and concomitant internal disorders. The patient became asymptomatic in a few weeks.

IgA pemphigus is a heterogeneous variant of pemphigus and must be differentiated from other blistering diseases including dermatitis herpetiformis, pemphigus herpetiformis, pemphigus foliaceus, pemphigus erythematosus, pemphigus vulgaris, pemphigus vegetans, paraneoplastic pemphigus or linear IgA bullous dermatosis. Particularly, IEN type typically shows greater clinical, histopathological and immunological heterogeneity than SPD type. Moreover, discrepancies between clinical, histopathological and immunological features made it difficult to finally establish the subtype of pemphigus IgA in some cases, including our patient.

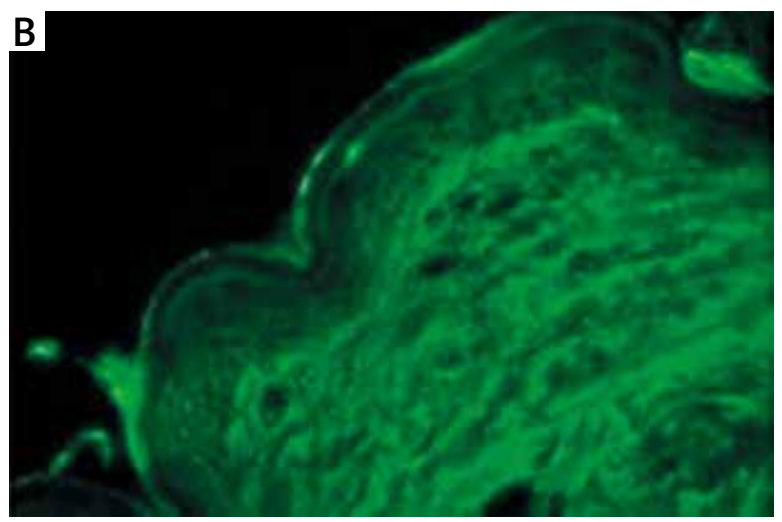

Figure 2. A - Histopathology of the skin lesion. Extensive neutrophilic pustules in the upper part of the epidermis and minimum pustules in the middle epidermis (hematoxylin and eosin stain), B - direct immunofluorescence of perilesional skin. IgA deposits on cell surfaces of the entire epidermis; C - indirect immunofluorescence of monkey esophagus. IgA anti-cell surface autoantibodies 


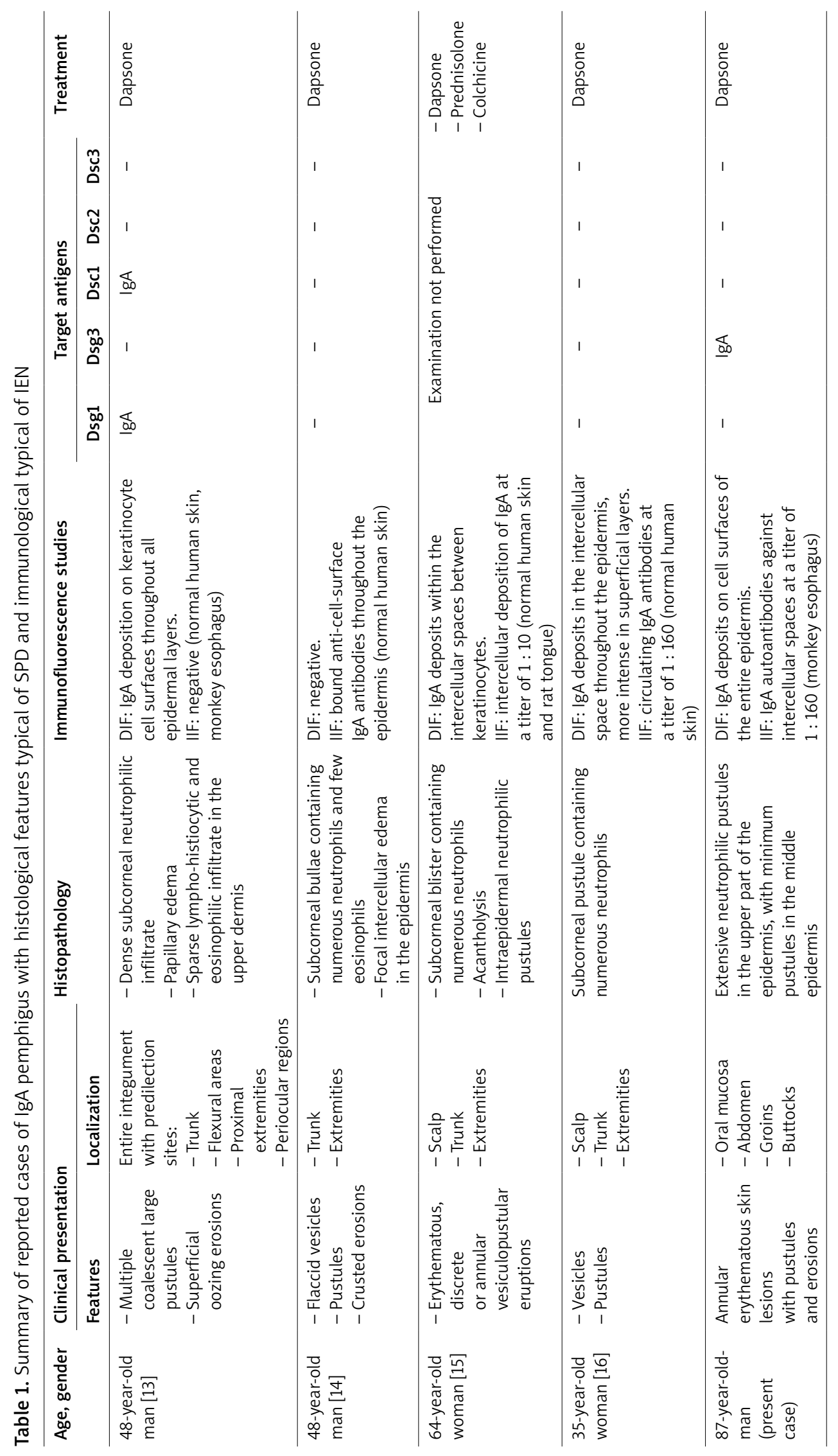


So far there have been described only four cases fulfilling histological features typical of SPD and immunological ones typical of IEN (Table 1). All of them clinically presented vesiculo-pustular eruptions localized in different areas such as the trunk, extremities, scalp and buttocks, whereas our patient showed superficial pustular lesions predominantly on the intertriginous areas, which were rather characteristic of SPD type IgA pemphigus [4-6]. Interestingly, the patient did not present deeper pustular lesions with sunflower-like configurations on the entire body usually observed in IEN type [4, 5]. However, our patient showed oral mucosal lesions that were not described in the four previously reported patients. In general, oral involvement is more frequent in cases of IEN type $[3,7]$ than SPD type.

Histopathology performed in the four cases disclosed neutrophilic infiltrates in the upper epidermis typically of SPD type [4]. However histopathology in our patient showed extensive pustules in the upper epidermis, but minimum pustules were also seen in the middle epidermis.

Direct immunofluorescence performed in the present case disclosed IgA deposits on cell surfaces of the entire epidermis, typically of IEN type [4, 5].

The discrepancy between histopathology features and DIF results encouraged us to perform antigen detection studies showing only ELISA with recombinant Dsg3 positive with circulating IgA antibodies. This result is along with the localization of IgA deposits in lower epidermis. Since our patient presented neutrophilic infiltrates mainly in the upper epidermis, whereas IgA deposits in the entire epidermis, it is likely that circulating IgA antibodies may be directed not only to Dsg3, but also to other antigens which were not detected in our system. Autoantigen detection studies showed negative results or were not performed in 3 of 4 cases. In one case circulating IgA antibodies reacted with Dsg1 and Dsc1 which differed from Dsg3.

In both subtypes of IgA pemphigus dapsone has been proved to be the first choice therapy $[6,8]$ usually at an effective dose of $100 \mathrm{mg} /$ day. Four previously reported cases and the present case were successfully treated with dapsone alone. However, various types of treatment have also been reported in IgA pemphigus like retinoids [9], systemic corticosteroids at moderate doses alone or in combination with immunosuppressive agents [6]. Rarely, azithromycin [10] or mycophenolate mofetil [11] may be used in intractable cases.

Recently Hashimoto et al. has proposed the term "intercellular IgA dermatosis (IAD)" for IgA pemphigus and classification dividing the disease into 6 subtypes [12]. Diagnosis of SPD-type and IEN-type was established for cases, which showed typical clinical and histopathological features. IgA-pemphigus vegetans (IgA-PVeg) was characterized by specific vegetating lesions with PVeglike histopathological presentation. If cases could not be categorized to these three subtypes they were deter- mined as unclassified IAD. Finally, diagnoses of IgA-pemphigus foliaceus (IgA-PF) and IgA-pemphigus vulgaris (IgA-PV) were based on immunological reactivity despite clinical and histopathological diagnoses of the 4 IAD subtypes. Cases with positive IgA ELISA results for Dsg1 were diagnosed as IgA-PF and for Dsg3 were diagnosed as IgAPV. Our patient presented discrepancies as clinical and histopathological features resembled SPD and immunological features were characteristic for IEN, according to the classification presence of anti-Dsg3 antibodies was essential. Eventually we consider our patient as IgA-PV.

\section{Conflict of interest}

The authors declare no conflict of interest.

\section{References}

1. Yasuda H, Kobayashi H, Hashimoto T, et al. Subcorneal pustular dermatosis type of IgA pemphigus: demonstration of autoantibodies to desmocollin-1 and clinical review. Br I Dermatol 2000; 143: 144-8.

2. Kárpáti S, Amagai M, Liu WL, et al. Identification of desmoglein 1 as autoantigen in a patient with intraepidermal neutrophilic IgA dermatosis type of IgA pemphigus. Exp Dermatol 2000; 9: 224-8.

3. Tajima M, Mitsuhashi Y, Irisawa R, et al. IgA pemphigus reacting exclusively to desmoglein 3. Eur J Dermatol 2010; 20: 626-9.

4. Robinson ND, Hashimoto T, Amagai M, Chan LS. The new pemphigus variants. J Am Acad Dermatol 1999; 40: 649-71.

5. Nishikawa T, Hashimoto T, Teraki Y, Ebihara T. The clinical and histopathological spectrum of IgA pemphigus. Clin Exp Dermatol 1991; 16: 401-2.

6. Wallach D. Intraepidermal IgA pustulosis. J Am Acad Dermatol 1992; 27: 993-1000.

7. Borradori L, Saada V, Rybojad M, et al. Oral intraepidermal IgA pustulosis and Crohn's disease. Br I Dermatol 1992; 126: 383-6.

8. Raap U, Völker B, Petering H, et al. Successful treatment of subcorneal pustular type of IgA pemphigus. Hautarzt 2005; 56: 66-70.

9. Gruss C, Zillikens D, Hashimoto T, et al. Rapid response of IgA pemphigus of subcorneal pustular dermatosis type to treatment with isotretinoin. J Am Acad Dermatol 2000; 43: 923-6.

10. Bliziotis I, Rafailidis P, Vergidis P, Falagas ME. Regression of subcorneal pustular dermatosis type of IgA pemphigus lesions with azithromycin. J Infect 2005; 51: E31-4.

11. Burchardt T, Büchau A, Ruzicka T, Megahed M. IgA pemphigus. Successful treatment with mycophenolate mofetil. Hautarzt 2004; 55: 387-9.

12. Hashimoto T, Teye K, Ishii N. Clinical and immunological studies of 49 cases of various types of intercellular IgA dermatosis and 13 cases of classical subcorneal pustular dermatosis examined at Kurume University. Br J Dermatol 2016 in press [Epub ahead of print].

13. Kopp T, Sitaru C, Pieczkowski F, et al. IgA pemphigus: occurrence of anti-desmocollin 1 and anti-desmoglein 1 antibody reactivity in an individual patient. J Dtsch Dermatol Ges 2006; 4: $1045-50$

14. lida K, Sueki H, Ohyama B, et al. A unique case of intra-epidermal neutrophilic dermatosis-type IgA pemphigus presenting with subcorneal pustules. Dermatology 2011; 222: 15-9. 
15. Kim SC, Won JH, Chung J, Bang DS. IgA pemphigus: report of a case with immunoelectron localization of bound IgA in the skin. J Am Acad Dermatol 1996; 34: 852-4.

16. Niimi Y, Kawana S, Kusunoki T. IgA pemphigus: a case report and its characteristic clinical features compared with subcorneal pustular dermatosis. J Am Acad Dermatol 2000; 43: 546-9. 